\title{
Tacrolimus Tópico nas Lesões Cutâneas Refratárias da Dermatomiosite Juvenil
}

\author{
Topical Tacrolimus in Refractory Skin \\ Lesions of Juvenile Dermatomiosytis
}

\author{
Carlos Nobre Rabelo Júnior ${ }^{(1)}$, Nádia Emi Aikawa ${ }^{(1)}$, Kátia Tomie Kozu ${ }^{(1)}$, Adriana Maluf Elias Sallum(2), \\ Lúcia Maria Arruda Campos ${ }^{(2)}$, Clovis Artur Almeida da Silva ${ }^{(3)}$
}

\section{RESUMO}

A dermatomiosite juvenil (DMJ) é uma doença inflamatória crônica idiopática que afeta principalmente músculos e pele. As lesões cutâneas podem persistir apesar do controle bem-sucedido da miosite. O tacrolimus tópico é um novo agente imunossupressor que tem sido usado no tratamento da dermatite atópica, com poucos relatos de seu uso em miopatias inflamatórias na faixa etária pediátrica. Foram descritos três pacientes com DMJ (dois meninos) com idade atual de 5,7 a 10,6 anos. A terapia inicial administrada para esses pacientes foi: corticosteróide (sistêmico em três e tópico em um), antimalárico em três e metotrexato em dois. Todos apresentavam lesões cutâneas refratárias (eritema malar, eritema difuso e/ou vasculite cutânea) após melhora significativa da fraqueza muscular. Tacrolimus tópico $0,03 \%$ foi usado duas vezes ao dia após falha do tratamento prévio. As características das lesões foram avaliadas de acordo com a extensão e a gravidade no início do uso do fármaco e após oito e 16 semanas. Na segunda avaliação (oito semanas), notavelmente um paciente apresentou melhora completa do eritema malar e do eritema difuso em membros e tronco, e dois tiveram melhora parcial do eritema malar e da vasculite cutânea. Na terceira avaliação (16 semanas), dois pacientes tiveram resolução completa das lesões e um apresentava eritema malar persistente e eritema moderado em glúteos e região lombar. No último paciente, a melhora das lesões cutâneas foi alcançada apenas após o uso de ciclosporina por 16 semanas. Nenhum deles teve efeitos adversos. Tacrolimus tópico pode ser considerado em pacientes com DMJ e manifestações cutâneas refratárias. Estudos posteriores controlados e randomizados com esse fármaco devem ser realizados nessa doença inflamatória.

Palavras-chave: tacrolimus tópico, dermatomiosite juvenil, envolvimento cutâneo.

\begin{abstract}
Juvenile dermatomyositis (JDM) is a rare idiopathic chronic inflammatory disease that affects mainly muscle and skin. Cutaneous lesions may persist despite successful treatment of myositis. Of note, topical tacrolimus is a new immunosuppressive agent that has been used to treat atopic dermatitis with few reports in pediatric inflammatory myopathies. Three JDM patients (two males) were described, current age from 5.7 to 10.6 years. The initial therapy administered for these patients were: corticosteroid (oral in three and topical in one), antimalarial in three and methotrexate in two. All of them had refractory skin lesions (malar rash, extensive rash and/or cutaneous vasculitis) after significant improvement of muscle weakness. Topical tacrolimus $0.03 \%$ was used twice daily after failure of previous treatment. The features of lesions were evaluated according to extension and severity at start of drug and after 8 and 16 weeks. At the second evaluation ( 8 weeks), remarkably one patient had complete improvement of malar rash and extensive rash in limbs and trunk, and two had partial improvement of malar rash and cutaneous vasculitis. At the third evaluation (16 weeks), two patients had complete resolution of lesions and one had persistent malar rash and moderate lumbar and gluteus rash. In the last patient, the improvement of skin lesions was reached only after 16 weeks of cyclosporine use. None of them had adverse effects. Topical tacrolimus could be considered in JDM patients with refractory cutaneous manifestations. Further randomized controlled trials with this agent should be performed in this inflammatory disease.
\end{abstract}

Keywords: topical tacrolimus, juvenile dermatomyositis, skin involvement.

\footnotetext{
Recebido em 02/08/07. Aprovado, após revisão, em 17/10/07. Declaramos a inexistência de conflitos de interesse.

Unidade de Reumatologia Pediátrica do Instituto da Criança do Hospital das Clínicas da Faculdade de Medicina da Universidade de São Paulo (ICr-HC-FMUSP).

1. Médico(a) da Complementação Especializada da Unidade de Reumatologia Pediátrica do ICr-HC-FMUSP.

2. Professora colaboradora do Departamento de Pediatria da FMUSP, doutora em Ciências pela FMUSP, médica-assistente da Unidade de Reumatologia

Pediátrica do ICr-HC-FMUSP.

3. Professor livre-docente do Departamento de Pediatria da FMUSP, médico responsável pela Unidade de Reumatologia Pediátrica do ICr-HC-FMUSP.

Endereço para correspondência: Clovis Artur Almeida da Silva, Rua Senador Cesar Lacerda Vergueiro, 494/82, Vila Madalena, CEP 05435-010, São Paulo, SP,

e-mail: clovisaas@icr.hcnet.usp.br
} 


\section{INTRODUÇÃO}

A dermatomiosite juvenil (DMJ) é uma doença sistêmica de etiologia desconhecida, caracterizada por vasculite de vasos de pequeno calibre ${ }^{(1,2)}$. Acomete preferencialmente pele e musculatura estriada esquelética podendo, entretanto, envolver outros órgãos e sistemas como trato gastrintestinal, pulmões e coração. Essa doença faz parte do grupo das miopatias inflamatórias idiopáticas, e é a mais comum na faixa etária pediátrica ${ }^{(3,4)}$. Nos Estados Unidos calcula-se uma incidência anual da doença de 3,2 por 1.000 .000 de crianças e adolescentes ${ }^{(5)}$. Por sua vez, estima-se que $16 \%$ a $20 \%$ dos pacientes adultos com dermatomiosite (DM) iniciam a doença na infância e na $\operatorname{adolescência~}^{(6)}$.

As alterações cutâneas patognomônicas da DMJ são heliotropo e pápulas de Gottron, que ocorrem em torno de $75 \%$ dos pacientes. Outras manifestações mucocutâneas incluem: fotossensibilidade, eritemas malar [semelhante ao do lúpus eritematoso sistêmico juvenil (LESJ)], e em outras áreas, eritema periungueal com anormalidades capilares, úlceras orais e cutâneas, hiperqueratose, calcinoses, além de manifestações vasculares, como fenômeno de Raynaud e telangiectasias ${ }^{(1,4)}$.

O tratamento farmacológico inicial da DMJ consiste em corticoterapia sistêmica associada aos antimaláricos a depender da gravidade do quadro cutâneo. Nos casos de doença resistente ou dependente da corticoterapia, podem-se utilizar imunossupressores, tais como: metotrexato, azatioprina, ciclofosfamida e ciclosporina e, menos comumente, imunoglobulina e agentes biológicos ${ }^{(7)}$. Até o presente momento não há estudos controlados na faixa etária pediátrica com tais medicamentos nessa doença.

A maioria dos pacientes tratados com medicações sistêmicas apresenta melhora importante do acometimento cutâneo da DMJ. Por sua vez, uma alternativa para os casos com lesões refratárias ao tratamento convencional é o uso de agentes tópicos. Destes, o tacrolimus (FK506) é um agente imunossupressor usado em afecções dermatológicas alérgicas, particularmente na dermatite atópica ${ }^{(8)}, \mathrm{e}$ constitui um tratamento adjuvante promissor em doenças auto-imunes $^{(9)}$, dentre elas a DM do adulto ${ }^{(10,11,12)}$. Até o presente momento há raras descrições da utilização desse medicamento tópico na $\mathrm{DMJ}^{(10)}$.

Assim sendo, os objetivos do presente estudo foram avaliar eficácia e eventos adversos do uso de tacrolimus tópico nos casos de DMJ refratários às terapias habituais.

\section{RELATO DOS CASOS}

No período de janeiro de 1983 a junho de 2007, foram atendidos 4.876 pacientes na Unidade de Reumatologia Pediátrica do ICr-HC-FMUSP, dentre os quais 141 (2,9\%) tiveram diagnóstico de $\mathrm{DMJ}$ de acordo com os critérios de Bohan e Peter ${ }^{(13)}$. Três destes $(2,1 \%)$ necessitaram do uso de tacrolimus tópico $0,03 \%$ e foram descritos, com avaliações sistematizadas no início da terapia, oito e 16 semanas após a introdução do fármaco.

Para avaliação objetiva da atividade clínica da doença, foi utilizado o Disease Activity Score (DAS). Este é um instrumento de mensuração da atividade clínica da DMJ que inclui os seguintes parâmetros: estado funcional, fraqueza muscular, tipo e distribuição do envolvimento cutâneo, vasculite e pápulas de Gottron. A pontuação varia de 0 a 20 de acordo com a inatividade ou atividade máxima da doença, respectivamente ${ }^{(14)}$.

A tabela 1 inclui os dados demográficos, a descrição das lesões cutâneas, a terapia prévia, a avaliação da eficácia e os eventos adversos.

\section{CASO 1}

Paciente de 10 anos, sexo masculino, iniciou seguimento na Unidade de Reumatologia Pediátrica do ICrHC-FMUSP em fevereiro de 2006. O quadro inicial em novembro de 2005 caracterizava-se por um expressivo envolvimento cutâneo (fotossensibilidade, heliotropo, pápulas de Gottron em interfalangeanas proximais bilaterais, eritema malar, eritemas palmar e periungueal, eritema difuso em tórax, abdômen e membros), associado à dor e à fraqueza muscular progressiva de predomínio proximal, disfonia e disfagia. $\mathrm{Na}$ investigação inicial, apresentava transaminase glutâmico oxalacética $(\mathrm{TGO})=858 \mathrm{U} / \mathrm{L}$ (valor normal: 10 a $36 \mathrm{U} / \mathrm{L})$, transaminase glutâmico pirúvica $(\mathrm{TGP})=906$ $\mathrm{U} / \mathrm{L}(24$ a $49 \mathrm{U} / \mathrm{L})$, creatinoquinase $(\mathrm{CK})=3.297 \mathrm{U} / \mathrm{L}$ $(39$ a $308 \mathrm{U} / \mathrm{L})$ e desidrogenase lática $(\mathrm{DHL})=2.197$ $\mathrm{U} / \mathrm{L}$ (141 a $231 \mathrm{U} / \mathrm{L})$. A eletroneuromiografia revelou padrão miopático e a biópsia muscular foi compatível com dermatomiosite. Em janeiro de 2006, após estabelecido o diagnóstico de DMJ, foi iniciada pulsoterapia com metilprednisolona (30 mg/kg/dia por três dias consecutivos), seguida por manutenção de prednisona $(2 \mathrm{mg} / \mathrm{kg} / \mathrm{dia})$ associada a difosfato de cloroquina $(5 \mathrm{mg} / \mathrm{kg} / \mathrm{dia})$. Evoluiu com rápida recuperação da força muscular e normalização dos parâmetros laboratoriais. Entretanto, em relação ao quadro cutâneo, persistiu com eritema malar e em regiões de tronco e membros, com melhora parcial de heliotropo 
TABELA 1

DADOS DEMOGRÁFICOS, DESCRIÇÃO DAS LESÕES CUTÂNEAS, TERAPIA PRÉVIA, AVALIAÇÃO DE EFICÁCIA E EVENTOS ADVERSOS EM TRÊS PACIENTES COM DERMATOMIOSITE JUVENIL QUE UTILIZARAM TACROLIMUS TÓPICO 0,03\%

\begin{tabular}{|c|c|c|c|}
\hline & Paciente 1 & Paciente 2 & Paciente 3 \\
\hline Idade ao diagnóstico (anos) & 9,4 & 3,2 & 7,1 \\
\hline Idade atual (anos) & 10,6 & 5,7 & 9 \\
\hline $\begin{array}{l}\text { Tempo entre diagnóstico e } \\
\text { introdução do tacrolimus (anos) }\end{array}$ & 0,8 & 2 & 1,2 \\
\hline Sexo & Masculino & Feminino & Masculino \\
\hline Tipo/localização das lesões & $\begin{array}{c}\text { Heliotropo, Gottron } \\
\text { Eritema malar intenso } \\
\text { Eritema em tórax, abdômen e membros } \\
\text { Eritema palmar e periungueal } \\
\text { Fotossensibilidade }\end{array}$ & $\begin{array}{l}\text { Heliotropo } \\
\text { Gottron } \\
\text { Eritema malar intenso } \\
\text { Eritema em tronco } \\
\text { Fotossensibilidade }\end{array}$ & $\begin{array}{c}\text { Heliotropo, Gottron } \\
\text { Eritema malar } \\
\text { Eritema em membros superiores, região } \\
\text { lombar, nádegas, coxas } \\
\text { Vasculites em face e polpas digitais } \\
\text { Fotossensibilidade }\end{array}$ \\
\hline Tratamentos prévios & $\begin{array}{c}\text { Corticosteróide sistêmico } \\
\text { antimalárico }\end{array}$ & $\begin{array}{c}\text { Corticosteróide sistêmico e tópico } \\
\text { antimalárico } \\
\text { Metotrexato }\end{array}$ & $\begin{array}{c}\text { Corticosteróide sistêmico } \\
\text { antimalárico } \\
\text { Metotrexato }\end{array}$ \\
\hline $\begin{array}{l}\text { Reavaliação } \\
8 \text { semanas } \\
16 \text { semanas }\end{array}$ & $\begin{array}{l}\text { Melhora completa } \\
\text { Melhora completa }\end{array}$ & $\begin{array}{l}\text { Melhora parcial } \\
\text { Melhora completa }\end{array}$ & $\begin{array}{l}\text { Melhora parcial } \\
\text { Ausência de resposta }\end{array}$ \\
\hline $\begin{array}{l}\text { DAS } \\
\text { Início } \\
8 \text { semanas } \\
16 \text { semanas }\end{array}$ & $\begin{array}{c}11 \\
2 \\
1\end{array}$ & $\begin{array}{l}7 \\
5 \\
1\end{array}$ & $\begin{array}{l}13 \\
12 \\
12\end{array}$ \\
\hline Eventos adversos & ausentes & ausentes & ausentes \\
\hline
\end{tabular}

e pápulas de Gottron. Em abril de 2006 iniciou-se redução gradual de prednisona e, em outubro de 2006, por manutenção de atividade cutânea (DAS = 11), foi introduzida pomada de tacrolimus $0,03 \%$ com duas aplicações diárias em face e tronco (dose de prednisona de $0,6 \mathrm{mg} / \mathrm{kg} / \mathrm{dia}$ na ocasião). A segunda avaliação foi realizada após oito semanas (dezembro de 2006), tendo sido evidenciada melhora importante das lesões eritematosas em face e tronco (DAS $=2$ ) com melhora mantida na terceira avaliação (após 16 semanas do medicamento tópico) (DAS = 1). A dose de prednisona foi progressivamente reduzida.

\section{CASO 2}

Paciente de 5 anos, sexo feminino, iniciou acompanhamento em julho de 2004 por mialgia, fraqueza muscular proximal e elevação de enzimas musculares associadas a pápulas de Gottron, heliotropo, eritema malar intenso, eritema em tronco e fotossensibilidade. Os exames iniciais evidenciavam: $\mathrm{TGO}=137 \mathrm{U} / \mathrm{L}(10$ a $36 \mathrm{U} / \mathrm{L}), \mathrm{TGP}=$ $100 \mathrm{U} / \mathrm{L}(24$ a $49 \mathrm{U} / \mathrm{L}), \mathrm{CK}=2.978 \mathrm{U} / \mathrm{L}(39$ a 308 $\mathrm{U} / \mathrm{L})$, aldolase $=30,5(<7,6 \mathrm{U} / \mathrm{L})$ e $\mathrm{DHL}=1.351 \mathrm{U} / \mathrm{L}$ (141 a $231 \mathrm{U} / \mathrm{L})$. A eletroneuromiografia foi compatível com miopatia de predomínio proximal e a biópsia muscular, sugestiva de miopatia inflamatória, indicando diagnóstico de DMJ. A paciente foi submetida a tratamento inicial com prednisona $(1,5 \mathrm{mg} / \mathrm{kg} /$ dia $)$ e sulfato de hidroxicloroquina $(5,5 \mathrm{mg} / \mathrm{kg} / \mathrm{dia})$. Após três meses havia apresentado melhora considerável da força muscular e diminuição do valor das enzimas musculares, porém mantinha hiperemia intensa de face e tronco e fotossensibilidade, sendo incluído metotrexato $(0,5 \mathrm{mg} / \mathrm{kg} / \mathrm{semana}$, por via oral $)$. Em um mês apresentou melhora significativa da atividade cutânea, sendo iniciada redução da dose do corticosteróide até sua suspensão em novembro de 2005. Em fevereiro de 2006 evoluiu com reativação das lesões cutâneas, com eritema malar intenso e piora das pápulas de Gottron e do heliotropo, e a dose de metotrexato foi aumentada para $0,8 \mathrm{mg} / \mathrm{kg} / \mathrm{semana}$, com administração subcutânea. Em junho de 2006 foi reintroduzida prednisona ( $1 \mathrm{mg} / \mathrm{kg} /$ dia) por recidiva do acometimento muscular. Nesta ocasião fez uso de creme de hidrocortisona $1 \%$ em face por 10 dias, sem resposta satisfatória (Figura 1). Em agosto de 2006 (DAS = 7) foi optado pela introdução de pomada de tacrolimus $0,03 \%$ com duas aplicações diárias em face e tronco e iniciada redução de prednisona em virtude do controle da atividade muscular. Após oito semanas de uso houve melhora parcial de eritema malar (DAS = 5) (Figura 2) e, em reavaliação posterior com 16 semanas, apresentava remissão da atividade cutânea em face (Figura 3) e nas demais localizações $(\mathrm{DAS}=1)$, possibilitando nova redução da dose de prednisona (dose atual de $0,5 \mathrm{mg} / \mathrm{kg} /$ dia). 


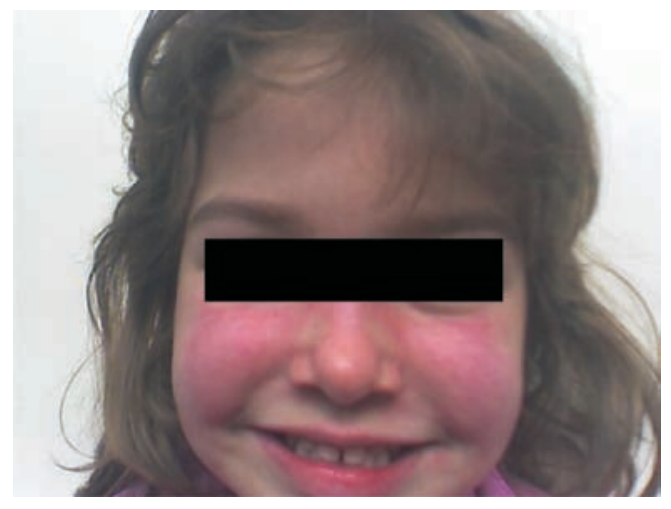

Figura 1 - Fotossensibilidade e eritema malar intenso antes do início do tratamento com tacrolimus tópico $0,03 \%$.

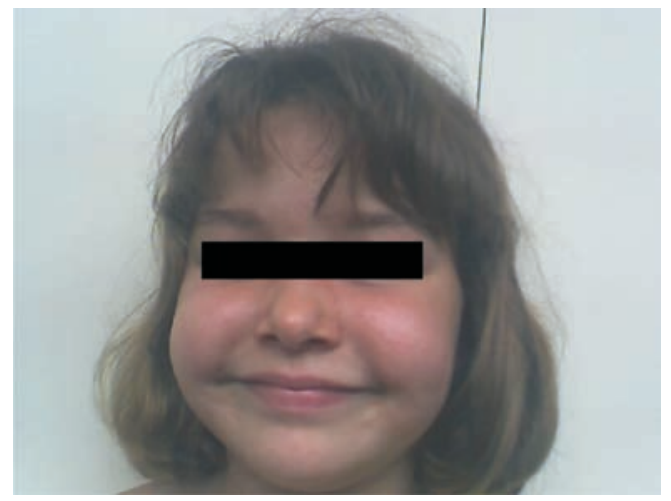

Figura 2 - Melhora parcial do eritema malar com oito semanas após tratamento com tacrolimus tópico 0,03\%.

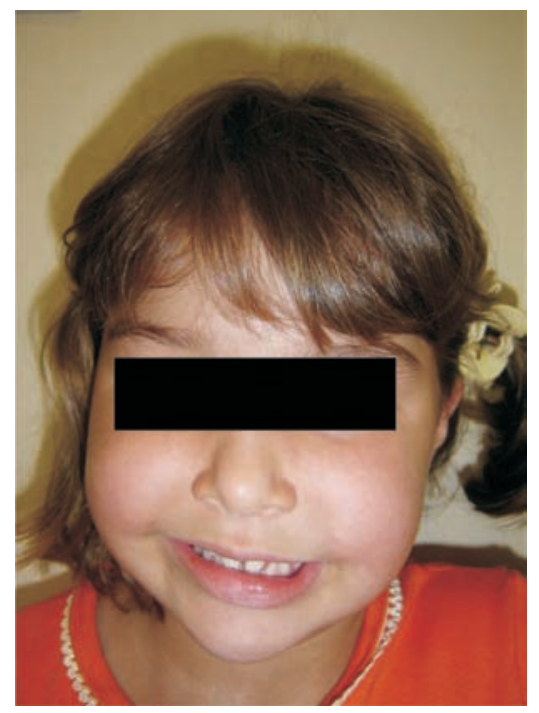

Figura 3 - Melhora completa do eritema malar 16 semanas após tratamento com tacrolimus tópico 0,03\%.

\section{CASO 3}

Paciente de 9 anos, sexo masculino, em janeiro de 2005 iniciou com poliartrite aguda associada a eritema malar e periorbitário, fadiga, dor e fraqueza muscular proximal. A investigação inicial revelou: TGO $=115 \mathrm{UI} / \mathrm{L}(10$ a 36 $\mathrm{U} / \mathrm{L}), \mathrm{TGP}=46 \mathrm{U} / \mathrm{L}(24$ a $49 \mathrm{U} / \mathrm{L}), \mathrm{CK}=752 \mathrm{U} / \mathrm{L}(39$ a $308 \mathrm{U} / \mathrm{l}), \mathrm{DHL}=622 \mathrm{U} / \mathrm{L}(141$ a $231 \mathrm{U} / \mathrm{L})$ e aldolase $=16,3 \mathrm{U} / \mathrm{L}(<7,6 \mathrm{U} / \mathrm{L})$. A eletroneuromiografia mostrou padrão miopático e a biópsia muscular foi compatível com dermatomiosite. Em abril de 2005 foi estabelecido o diagnóstico de DMJ, mantida prednisona $0,7 \mathrm{mg} / \mathrm{kg} / \mathrm{dia}$ (em uso há dois meses) e associados fotoproteção e difosfato de cloroquina $(4,5 \mathrm{mg} / \mathrm{kg} / \mathrm{dia})$. As manifestações cutâneas foram exuberantes: heliotropo, pápulas de Gottron em interfalangeanas proximais bilaterais, cotovelos e joelhos, eritema malar intenso com lesões vasculíticas em face e periungueais, eritema descamativo importante em região lombar (maior à direita), nos braços, nas coxas e nas nádegas. Em junho de 2005 foi introduzido metotrexato $(0,4 \mathrm{mg}$ / $\mathrm{kg} / \mathrm{sem}$, via oral) e aumentada gradativamente a dose (até $1 \mathrm{mg} / \mathrm{kg} / \mathrm{sem}$, via subcutânea) por persistência de atividade clínico-laboratorial da doença, no período de seis meses. Concomitantemente, aumentou-se a dose de prednisona até $1 \mathrm{mg} / \mathrm{kg} /$ dia, havendo assim recuperação da força muscular e diminuição do valor das enzimas musculares. Em janeiro de 2006 foi optado por redução gradual da corticoterapia com sua suspensão em dezembro de 2006 em virtude de complicações de seu uso a longo prazo (catarata bilateral, osteoporose e fratura vertebral). Durante todo esse período houve persistência das lesões cutâneas a despeito da melhora do comprometimento muscular. Em março de 2006 foi aumentada a dose da cloroquina $(6 \mathrm{mg} / \mathrm{kg} /$ dia $)$ na tentativa de controlar o quadro cutâneo (em associação com metotrexato em dose máxima e fotoproteção) sem, entretanto, resposta satisfatória. Em junho de 2006 optou-se pela introdução do tacrolimus tópico $0,03 \%$, duas vezes ao dia (DAS = 13). $\mathrm{Na}$ primeira reavaliação (oito semanas) havia tido melhora discreta das lesões em região lombar, nas nádegas e nas coxas, sem nenhuma alteração das lesões em face e braços (DAS = 12). Na segunda reavaliação (16 semanas) não houve qualquer alteração em relação à anterior (DAS = 12). Assim sendo, em outubro de 2006 foi associada ciclosporina pela gravidade do quadro cutâneo na dose de $3,5 \mathrm{mg} / \mathrm{kg} /$ dia e aumentada a dose até $5 \mathrm{mg} / \mathrm{kg} /$ dia em quatro meses. $\mathrm{Na}$ última consulta (fevereiro de 2007), o paciente apresentava melhora parcial das lesões cutâneas, com diminuição considerável do eritema em face, braços, região lombar, nádegas e coxas e heliotropo residual. 


\section{DISCUSSÃO}

Na Unidade de Reumatologia Pediátrica do ICr-HCFMUSP, a terapia inicial da vasculite cutânea da DMJ é geralmente realizada com antimaláricos, corticosteróide sistêmico e, ocasionalmente, corticosteróide tópico. Nos casos refratários às terapias anteriores, têm sido indicados imunossupressores (como metotrexato e/ou ciclosporina) ou gamaglobulina endovenosa. Mais raramente são usadas talidomida e dapsona. Além disto, imunossupressores tópicos têm sido utilizados no manejo da doença. O presente estudo foi um dos poucos relatos da literatura ${ }^{(10)} \operatorname{com}$ a utilização do tacrolimus tópico $0,03 \%$ em crianças e adolescentes com DMJ e demonstrou que este foi um medicamento seguro e eficaz, adjuvante ao tratamento sistêmico (corticoterapia, antimaláricos e imunossupressores) da doença.

Um aspecto relevante das lesões cutâneas da DM do adulto é o impacto na qualidade de vida dos pacientes, resultando em desconforto físico e estresse emocional ${ }^{(15,16)}$. Tal fato indiscutivelmente é prioritário na população de escolares e adolescentes, uma vez que a imagem corporal é um fator de grande importância na auto-estima desses grupos.

O tacrolimus é um agente imunossupressor produzido pelo fungo Streptomyces tsukubaensis que atua inibindo a ativação de linfócitos T e a transcrição de IL-2 e de outras citocinas $^{(17)}$.

Em 2000, este foi inicialmente aprovado pela US Food and Drugs Admnistration (FDA) com segurança e eficácia comprovada para dermatite atópica ${ }^{(18)}$. Esse medicamento foi também utilizado em doenças auto-imunes, tais como: pioderma gangrenoso, psoríase, doença do enxerto- versushospedeiro, alopecia areata, lúpus eritematoso sistêmico e doença de Behçet ${ }^{(9,19)}$. Além disso, seu uso tem sido descrito em outras afecções auto-imunes com comprometimento dermatológico refratário às terapias habituais ou que apresentaram eventos adversos aos corticosteróides e imunossupressores (osteoporose, catarata, hipertensão arterial, infecções, toxicidade renal e hepática, dentre outros), como DM do adulto e DM amiopática, a maioria com bons resultados ${ }^{(12,19,20)}$.

Até o presente momento, há 15 casos relatados em DM do adulto, três em DMJ e dois casos em DM amiopática (um destes, adolescente) que receberam tacrolimus tópico entre $0,03 \%$ e $0,1 \%$. Destes, melhora completa ocorreu em sete, parcial em quatro e ausência de resposta ao fármaco em nove. A má resposta observada pode ser atribuída à sua utilização em lesões crônicas e hiperqueratóticas, com baixa absorção cutânea da medicação ${ }^{(10,21)}$.

Neste estudo utilizou-se o período de oito semanas para a reavaliação dos pacientes, intervalo este empregado pela maioria dos casos relatados ${ }^{(12,20)}$. Uma nova consulta foi realizada após 16 semanas com o intuito de reavaliar a manutenção da resposta inicial. Em virtude da disponibilidade no presente serviço, foi utilizada pomada de tacrolimus $0,03 \%$, diferente da maioria dos artigos publicados ${ }^{(19,20)}$, os quais optaram pela concentração de $0,1 \%$. Possivelmente a menor potência imunossupressora pode ter interferido na resposta terapêutica, especialmente no caso 3 , em que o comprometimento cutâneo era mais grave.

O tacrolimus tópico $0,03 \%$ foi mais precocemente introduzido no caso 1 , tendo-se observado uma melhora rápida e sustentada em relação aos outros dois pacientes. Nos casos 2 e 3, o uso adicional de terapia imunossupressora (metotrexato) não resultou em melhora significativa do comprometimento cutâneo. Tais evidências indicam que o uso precoce da medicação tópica deve ser considerado como uma alternativa, antes mesmo da introdução de agentes sistêmicos com efeitos potencialmente deletérios.

Eventos adversos não foram observados com tacrolimus tópico $0,03 \%$ em nenhum dos três casos relatados. $\mathrm{Na}$ literatura médica, prurido, ardor e eritema têm sido descritos ${ }^{(9)}$. Entretanto, telangiectasias e atrofia cutânea, efeitos comuns dos corticosteróides tópicos, não ocorrem com o tacrolimus tópico $0,03 \%{ }^{(22)}$.

Este estudo reforça a segurança e a eficácia do tacrolimus tópico como alternativa nas lesões cutâneas da DMJ, refratárias à terapia-padrão, particularmente quando introduzido precocemente no curso da doença. Em virtude de pequena casuística deste estudo, faz-se necessária a realização de estudos controlados com populações maiores e a utilização de instrumentos voltados especificamente para avaliação do comprometimento cutâneo da DMJ. 


\section{REFERÊNCIAS}

1. Sallum AM, Kiss MH, Sachetti S, et al.: Juvenile dermatomyositis: clinical, laboratorial, histological, therapeutical and evolutive parameters of 35 patients. Arq Neuropsiquiatr 60: 889-99, 2002.

2. Sallum AM, Marie SK, Wakamatsu A, et al.: Immunohistochemical analysis of adhesion molecule expression on muscle biopsy specimens from patients with juvenile dermatomyositis. J Rheumatol 31: 801-7, 2004.

3. Sallum AM, Kiss MH, Silva CA, et al.: Difference in adhesion molecule expression (ICAM-1 and VCAM-1) in juvenile and adult dermatomyositis, polymyositis and inclusion body myositis. Autoimmun Rev 5: 93-100, 2006.

4. Nukumizu LA, Liphaus BL, Barros PC, Sallum AM, Silva CA: Associação de dermatomiosite juvenil e lúpus eritematoso sistêmico juvenil. Rev Bras Reumatol 42: 407-10, 2002.

5. Mendez EP, Lipton R, Ramsey-Goldman R, et al.: US incidence of juvenile dermatomyositis, 1995-1998: results from the National Institute of Arthritis and Musculoskeletal and Skin Diseases Registry. Arthritis Rheum 49: 300-5, 2003.

6. Medsger TA, Dawson WN, Masi AT: The epidemiology of polymyositis. Am J Med 48: 715-23, 1970.

7. Stringer E, Feldman BM: Advances in the treatment of juvenile dermatomyositis. Curr Opin Rheumatol 18: 503-6, 2006.

8. Kang S, Lucky AW, Pariser D, Lawrence I, Hanifin JM: Longterm safety and efficacy of tacrolimus ointment for the treatment of atopic dermatitis in children. J Am Acad Dermatol 44: 58-64, 2001 .

9. Zabawski EJ, Costner M, Cohen JB, Cockerell CJ: Tacrolimus: pharmacology and therapeutic uses in dermatology. Int J Dermatol 39: 721-7, 2000.

10. Yoshimasu T, Ohtani T, Sakamoto T, Oshima A, Furukawa F: Topical FK506 (tacrolimus) therapy for facial erythematous lesions of cutaneous lupus erythematosus and dermatomyositis. Eur J Dermatol 12: 50-2, 2002.
11. Ueda M, Makinodan R, Matsumura M, Ichihashi M: Successful treatment of amyopathic dermatomyositis with topical tacrolimus. Br J Dermatol 148: 595-6, 2003.

12. Peyrot I, Sparsa A, Loustaud-Ratti V, et al.: Topical tacrolimus and resistant skin lesions of dermatomyositis. Rev Med interne 27: 730-5, 2006

13. Bohan A, Peter JB: Polymyositis and dermatomyositis (first of two parts). N Engl J Med 292: 344-7, 1975.

14. Bode RK, Klein-Gitelman MS, Miller ML, Lechman TS, Pachman LM: Disease activity score for children with juvenile dermatomyositis: reliability and validity evidence. Arthritis Rheum 49: 7-15, 2003.

15. Jayaprakasam A, Darvay A, Osborne G, McGibbon D: Comparison of assessments of severity and quality of life in cutaneous disease. Clin Exp Dermatol 27: 306-8, 2002.

16. Hundley JL, Carroll CL, Lang W, et al.: Cutaneous symptoms of dermatomyositis significantly impact patients' quality of life. J Am Acad Dermatol 54: 217-20, 2006.

17. Sawada S, Suzuki G, Kawase Y, Takaku F: Novel immunossuppresive agent, FK506. In vitro effects on the cloned T-cell activation. J Immunol 139: 1797-803, 1987.

18. US FDA: US FDA Advisory Committee recommends approval for tacrolimus ointment. Skin Therapy Letter 6: 5, 2000.

19. Lampropoulos E, Cruz P: Topical tacrolimus treatment in a patient with dermatomyositis. Ann Rheum Dis 64: 1376-7, 2005.

20. Hollar CB, Jorizzo JL: Topical tacrolimus $0.1 \%$ ointment for refractory skin disease in dermatomyositis: a pilot study. J Dermatolog Treat 15: 35-9, 2004.

21. García-Doval I, Cruces M: Topical tacrolimus in cutaneous lesions of dermatomyositis: lack of effect in side-by-side comparison in five patients. Dermatology 209: 247-8, 2004.

22. Reitamo S, Rissanen J, Remitz A: Tacrolimus ointment does not affect collagen synthesis: results of a single-center randomized trial. J Invest Dermatol 111: 396-8, 1998. 